\title{
Fluorescence Assisted Cancer Surgery
}

\author{
Obipiseibima P Aggokabo ${ }^{1 *}$ and Raymond Donbezier Fatchu ${ }^{2}$ \\ ${ }^{1}$ Department of Sports and Exercise Science, Liverpool John Moores University, UK \\ ${ }^{2}$ Pathology Division, 37 Military Hospital, Ghana
}

*Corresponding author: Obipiseibima P Aggokabo, Department of Sports and Exercise Science, Liverpool John Moores University, UK.

To Cite This Article: Obipiseibima P Aggokabo, Raymond Donbezier Fatchu. Fluorescence Assisted Cancer Surgery. 2020 - 11(1). AJBSR. MS.ID.001589. DOI: 10.34297/AJBSR.2020.11.001589.

Received: 畊 November 23, 2020; Published: 眥 November 30, 2020

\section{Introduction}

The surgical procedure involves the cutting into patient tissue as part of disease management. Surgical resection therefore is a critical modality for disease management and treatment. The essence of the surgical process is to remove as safely as possible as much cancer tissue as possible. This is to a large extent dependent on the surgeon's ability to clearly distinguish cancer tissue from normal tissue, remove as much cancer tissue as possible while preserving normal tissue [1]. Unfortunately, this ability is based on local visualization of the tumour and/or the ability to palpate it [2].

On the contrary, fluorescence assisted cancer surgery is used to guide defining tumour locations and margins during surgical procedures. This allow for a more complete resection of a tumour, avoid unnecessary damage to normal tissue and decrease the need of repeat surgery for missed tumours [3]. Fluorescent indicators had been a well-established tool that serves in the monitoring of various concentrations of physiologically important ions within living cells [4,5]. These fluorescent indicators mostly respond with various changes in fluorescence or with varying shifts in emission or excitation spectra.

However, fluorescent techniques indicators can emit information on the dynamic process on a nanosecond timescale. Recent imaging strategies commonly used for both cancer detection and pre-treatment planning respectively, are based on the anatomical or the metabolically morphology in the tissue [6]. Fluorescence techniques provide considerable imaging for distinguishing between tumour and normal tissue. The use of ionizing radiation in these techniques ensures that there is real time anatomical and functional imaging.

The field of fluorescence assisted surgeries has seen tremendous growth in research and practice. In several surgical procedures in recent years, the practice of fluorescence assisted surgery has played significant roles during surgery and in procedures such as the identification of solid tumours, sentinel lymph node mapping, lymphography, anatomical imaging and angiography [7]. Most importantly the procedure can be used without having significant interruptions to the workflow of the surgeon.

The fluorescence assisted cancer surgery technique, aids in the anatomical signature of various tumour as can be viewed through the use of radiographic imaging or ultrasound providing the ability to differentiate a normal cell from a tumour while the main disadvantage of this technique is the limited spatial resolution involved and the difficulty of both translating a two-dimensional information to a three-dimensional surgical field [8]. The fluorescence assisted cancer surgery technique is safe as it makes use of non-ionizing radiation and moreover, this technique enables real-time anatomical and functional imaging.

\section{Principles of Fluorescent Indicators for Cancer Surgery}

The process of fluorescence imaging was first introduced in surgery in the year 1948 when surgeons used the intravenous fluorescent dye, fluorescein, to improve upon intracranial neoplasm detection during surgery [9]. The fluorescent agents have been reportedly used for several other surgical procedures. In fluorescence assisted surgery, source light is directed through the tissue from a light filter with low intensity. The filtered light is absorbed by fluorophores, which may be either endogenous (autofluorsecent) or exogenous (injected fluorescein) [10]. The fluorophores are energised to an excitation state where they reemit photons to a ground state [11]. The re-emission photons are then detected with a charged couple device that can generate a colour and fluorescence imaging [11]. 


\section{Difference in Metabolism Between Cancer Cells and Normal Cells}

Clinically, fluorescent agents are known for its importance in the establishment of positive margins with a high resolution, map lymph nodes, detection of residual disease, prediction of metastatic potential and in the process of drug delivery [12-15] The metabolism of cancer is characterised by an uptake of glucose utilisation [12-16]. However, in a normal cell, glucose is been catabolised to pyruvate. Through the mitochondrial tricarboxylic cycle (TCA) pyruvzate is further converted to acetyl CoA with further oxidation to carbon dioxide. This leads to the production of Nicotinamide Adenine dinucleotide (NADH) and Flavin Adenine Dinucleotide (FADH)2. The process of glycolysis, TCA cycle and the electron transfer phosphorylation results in the transfer of electrons from NADH and FADH2 to oxygen to produce 36 Adenosine Triphosphate (ATP) molecules per glucose molecule.

Despite the abundance of oxygen, cancers cells preferentially utilise glycolysis as a preferred pathway as compared to normal cells which do when oxygen supply is limited $[17,18]$. The production of lactate because of the increased glucose uptake even under aerobic conditions is what is known as the Warburg effect [19].

Cancer cells are therefore compelled to follow the glycolytic pathway instead of the oxidative phosphorylation pathway. This metabolic change has been hypothesised to result in the damage to the mitochondrial oxidative phosphorylation process (Figure 1).

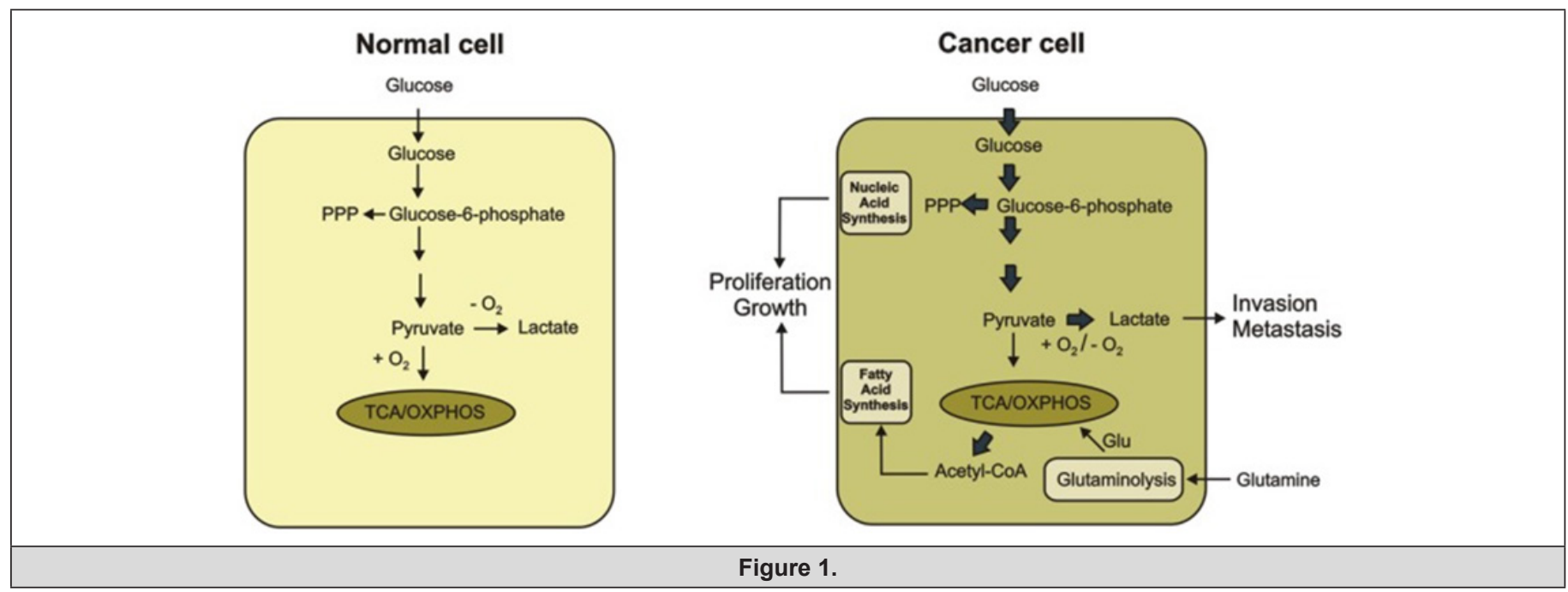

This figure shows the metabolic difference between the normal cells and cancer cells. In a normal cell, the glucose is metabolized to pyruvate which is oxidised in the presence of carbon dioxide through the TCA cycle and oxidative phosphorylation process in the mitochondria. The cancer converts glucose to lactate in the presence or absence of 02 . Glutamine is then produced which serves as a major substrate to refuel the TCA cycle [20-22]. An increase in the synthesis of nucleic acid and fatty acid (lipids), promotes the proliferation and growth of cancer cells.

\section{Indicator Used in Fluorescence Assisted Cancer Surgery for Glioma}

One of the indicators used in fluorescence assisted cancer surgery for glioma is the 5-aminolevulinic acid (5-ALA). This is a non-fluorescent pro-drug which leads to the absorption of fluorescent porphyrins with malignant tumour cells. The patients ingest the 5-ALA before a surgical procedure to enhance radical resection. This is administered intravenously or orally prior to craniotomy to glioblastoma patients. 5-ALA is taken up by the highgrade glioma cells, causing the cells to fluorescence pink when viewed under blue light, thus increasing the visibility of the tumour resection [23]. This procedure can be done with an exoscope, a system that consist of a tubular telescope connected to a camera and a high definition monitor [24].

This technique is achieved by implementing a system to switch between white and blue light and installing an observation filter $(440 \mathrm{~nm})$ in between the surgical field and the microscope. However, the fluorescence assisted cancer surgery technique for the detection of glioma is limited by many factors which solely depends on the fluorescent agent used.

\section{Antibody Targeting Specific Biomarkers on The Surface of The Cancer Cells}

Anti-CD133, anti-CD44 and anti-EpCAM are the various antibody targeting specific biomarkers on the surface of cancer cells. The commonly used cancer stem cells (CSC) biomarkers are the anti-CD44 is a cell surface with an extracellular matrix receptor [25]. The CD44 antibody serves as a major anti-CSC approach and studies by Deonarain et al. [26] has shown that the H90, an antiCD44 therapy successfully eradicated the acute myeloid leukaemia [26]. 
The CD133, a transmembrane glycoprotein and a CSC biomarker found in several tumours such as glioblastoma, colon and hepatocellular cancers. Also, the CD133 CSCs, have consistently shown resistance to both radiotherapy and chemotherapy because of their higher expression of deoxyribonucleic acid repair, lower proliferation, and anti-apoptotic gene $[27,28]$. The EpCAM has been discovered as CSC marker in solid tumours and humanised EpCAM antibodies have been successful in both preclinical and early clinical studies showing potent anti-tumour activity [26,29].

\section{Importance of Fluorescence Assisted Cancer Surgery}

During oncologic surgeries it becomes necessary for the surgery to clearly determine in real time the margins of a tumour to enable complete resection of the cancerous tissue. Current surgical procedures allow for the analysis of frozen sections. Unfortunately, this procedure is time consuming and is reported to account for discrepancies in $5-15 \%$ of cases [2,10]. Fluorescence guided surgery however provides real time guided imagery of tumours during surgery and offers a more objective assessment of intraoperative surgical margins [1] when compared to other procedures such as frozen sections. Also, an improved visualization of the cancer can reduce damage to important normal structures such as nerves, blood vessels, ureters, and bile ducts.

Some desirable characteristics of real time fluorescence assisted surgery are described in Table 1.

Table 1: Desirable characteristics of real time fluorescence assisted surgery. Table adapted from [30].

\begin{tabular}{|c|c|}
\hline Characteristic & Description \\
\hline Intra-Operative & Less than or equal to 24p frames per second image acquisition and display \\
\hline Real Time & Indicates a varying contrast agent that specifically accumulates in the tumour being imaged. \\
\hline High Level of Specificity & Detection of low fluorescence signal and small tumours \\
\hline High Level of Sensitivity & Ability to detect small tumours and tumour boundary \\
\hline High Resolution & Ergonomically sound and hands-free \\
\hline It is Wearable & Accurate information is displayed in a form that does not serve as a distraction to the surgeon during surgery. \\
\hline Non-Interfering & Requires minimal and non-specialised training \\
\hline It is User Friendly & \\
\hline
\end{tabular}

The Importance of The Fluorescence Assisted Cancer Surgery Technique in Surgery

Surgery has been known as a primary mode for the treatment of various malignancies and the aim of surgery is to safely remove as much cancer cells from a patient. The recent invention of the optical imaging with fluorescence assisted surgery, is gradually bridging the gap between the real-time tumour specific identification [31].

Although, the fluorescence assisted cancer surgery technique is currently being applied to a wide range of tumour types and has the potential to be of great impact in surgical practice and cancer surgery [32]. This remains unclear which disease population will have the greatest benefit from use of this technology.

\section{Challenges}

Though the applications of fluorescence assisted surgery has several reported benefits it is not without challenges. According to a publication by Mark J Landau [7] in the Annals of Translational Medicine, there may be some portion a population who will be sensitive to some probes such as Iodocyanine Green (ICG) and would therefore require alternative probes. This therefore implies that the sensitivity of patients to any designed probe would have to be tested prior to the administration of the probe.

It is important to acknowledge the challenge of identification of metastasized tissue even in the presence of fluorescent probes. Very limited studies have been identified which evaluate how metastasized cells of a localized tumour would be identified. For instance, in identifying the margins of a prostate tumour, the fluorescent probe would be administered close to the tumour location. This would ensure the clear visualization of the tumour margins and ensure the complete resection of the tumour. However, in the event where the tumour may have metastasized to other locations such as the lymph nodes of the lungs, the use of the probe would have missed the metastasized cancer tissue.

The frontiers of oncology argue that fluorescence guided surgery is less expensive compared to traditional optical procedures [2]. While this may seem true it is important to note that there would very high costs in the design of the fluorescent probes in addition to some further costs in the approval process. Fluorescent probes may also have to be designed for person specific conditions where such patients are sensitive or may not present the required ligands for a generic probe. This essentially would not make the procedure as less expensive as expected.

One other area which may have seen limited studies is the evaluation of the side effects of the probes, if any, and the clearance time of the fluorescent probe from the body system after administration.

\section{Practical Problems in Applying the Fluorescent Assisted Cancer Surgery Technique}

Fluorescence assisted cancer surgery technique, has been useful and productive particularly in the diagnosis and removal 
of brain $[23,33]$ bladder and head and neck tumours, where the tumour is located at a target organ site. However, the application of the current fluorescence technology to several forms of cancers such as sarcoma is mainly prevented due to a complete resection without direct visualisation of the mass.

Fluorescence assisted cancer surgery enhances tumour detection, margin confirmation, surgical navigation and in most cases can be combined with therapeutic techniques for the elimination of microscopic disease [34]. This technique can help in the detection of lesions and prevent unwanted and futile surgeries in the presence of metastatic diseases.

\section{Future development of fluorescence assisted cancer surgery}

Fluorescence assisted cancer surgery technique has been used to achieve a wide range of preliminary success [35] and achieved some clinical success. However, this technique may lead to the improvement of tumour resection rates while minimizing tissue resection [36] and this can translate effectively into more improved clinical outcomes.

Several techniques like the Intraoperative fluorescence imaging, used in fluorescence assisted cancer surgery are emerging that selectively irradiate cancer cells, creating a distinction between various tumours and surrounding tissues with the prospective for single-cell sensitivity.

\section{Conclusion}

Surgery can be said to be the most definite approach to treating solid tumours. However, the surgical process can be expensive, time consuming and fatal. It is therefore necessary that the surgical process meets its complete purpose to avoid the need of a repeat surgery for the same earlier purpose. Fluorescence guided surgery ensures a more definite and clear definition of surgical margins for effective navigation and complete resection of tumours.

It is important however, that advances in the procedure further focusses on the identification of metastasized tumours to ensure all such tumours are removed in addition to the localized or primary cancer cells.

\section{Reference}

1. Mondal SB, Shengkui Gao, Nan Zhu, Rongguang Liang, Viktor Gruev, et al. (2014) Real-time fluorescence image-guided oncologic surgery. Adv cancer res 124: 171-211.

2. Nagaya, AN, Peter L Choyke, Hisataka Kobayashi (2017) FluorescenceGuided Surgery. Front Oncol 7: 314.

3. Thinzar M Lwin, R M H, M B (2018)) The development of fluorescence guided surgery for pancreatic cancer: from bench to clinic Expert Rev Anticancer Ther 18(7): 651-662.

4. Demchenko AP (2009) Introduction to fluorescence sensing. Springer, Amsterdam, USA
5. Lakowicz JR (2006) Principles of fluorescence spectroscopy. Springer, NewYork, USA.

6. Hricak, $\mathrm{H}$ (2005) MR imaging and MR spectroscopic imaging in the pretreatment evaluation of prostate cancer. Br J Radiol 2: S103-S111.

7. Mark J Landau, DJG, Ketan M Patel (2016) Advances in fluorescent-image guided surgery. Ann Trans Med 4(20): 392.

8. Quyen T Nguyen, Emilia S Olson, Todd A Aguilera, Tao Jiang, Miriam Scadeng, et al. (2010) Surgery with molecular fluorescence imaging using activatable cell-penetrating peptides decreases residual cancer and improves survival. Proc Natl Acad Sci U S A 107(9): 4317-4322.

9. Acerbi F, Claudio Cavallo, Morgan Broggi, Roberto Cordella, Elena Anghileri, et al. (2014) Fluorescein-guided surgery for malignant gliomas: a review. Neurosurg Rev 37(4): 547-557.

10. Rosenthal EL, WJ de Boer E, Basilion JP, Biel MA, Bogyo M, et al. (2016) Successful translation of fluorescence navigation during oncologic surgery: a consensus report. J Nucl Med 57(1): 144-150

11. de Boer E, N J Harlaar, A Taruttis, W B Nagengast, E L Rosenthal, et al. (2015) Optical innovations in surgery. Br J Surg 102 (2): e56-72.

12. Chongwei Chi, Yang Du, Jinzuo Ye, Deqiang Kou, Jingdan Qiu, et al. (2014;) Intraoperative imaging-guided cancer surgery: from current fluorescence molecular imaging methods to future multi-modality imaging technology. Theranostics 4(11): 1072-1084.

13. Hussain T, NQ (2014) Molecular imaging for cancer diagnosis and surgery. Adv Drug Deliv Rev 66: 90-100.

14. Kim JC, Jong L Lee, Yong S Yoon, Abdulrahman M Alotaibi, Jihun Kim (2016) Utility of indocyanine-green, fluorescent imaging during robotassisted sphincter-saving surgery on rectal cancer patients. Int J Med Robot 12(4): 710-717.

15. Juliette Mérian, Julien Gravier, Fabrice Navarro, Isabelle Texier (2012) Fluorescent nanoprobes dedicated to in vivo imaging: from preclinical validations to clinical translation. Molecules 17(5): 5564-5591.

16. Ryan K Orosco, Roger Y Tsien, Quyen T Nguyen (2013) Fluorescence imaging in surgery. IEEE Rev Biomed Eng 6: 178-187.

17. DeBerardinis RJ, L J, Hatzivassiliou G, Thompson CB (2008) ) The biology of cancer: metabolic reprogramming fuels cell growth and proliferation. Cell Metab 7(1): 11-20.

18. Phan LM, Sai CJY, Lee HM (2014) Cancer metabolic reprogramming: importance, main features, and potentials for precise targeted anticancer therapies. Cancer Biol Med 11(1): 1-19.

19. Vander Heiden MG, CL, Thompson CB (2009)) Understanding the Warburg effect: the metabolic requirements of cell proliferation. Science 324(5930): 1029-1033.

20. Kato Y, LC, Colige AC, Mineur P, Noël A, et al. (2005) Acidic extracellular $\mathrm{pH}$ induces matrix metalloproteinase- 9 expression in mouse metastatic melanoma cells through the phospholipase D-mitogen-activated protein kinase signaling. J Biol Chem 280(12) :10938-10944.

21. Bonuccelli G, TA, Whitaker Menezes D, Pavlides S, Pestell RG, et al. (2010) Ketones and lactate "fuel" tumor growth and metastasis: evidence that epithelial cancer cells use oxidative mitochondrial metabolism. Cell Cycle 9(17): 3506-3514.

22. Martinez Outschoorn UE, PM, Ertel A, Tsirigos A, Lin Z, et al. (2011) ) Ketones and lactate increase cancer cell "stemness," driving recur-rence, metastasis and poor clinical outcome in breast cancer: achieving personalized medicine via metabolo-genomics. Cell Cycle 10(8): 12711286.

23. Stummer W, PU, Meinel T, Wiestler OD, Zanella F, et al. (2006) Fluorescence-guided surgery with 5 -aminolevulinic acid for resection of 
malignant glioma: a randomised controlled multicentre phase III trial Lancet Oncol 7(5): 392-401.

24. Aleksandr A Potapova, Dmitry J Usacheva, Valery A Loshakova, Vasilii A Cherekaeva, Valery N Kornienko, et al. (2008) First experience in 5-ALA fluorescence-guided and endoscopically assisted microsurgery of brain tumors. Medical Laser Application 23(4): 202-208.

25. Chen, K Ying hui Huang, Ji long Chen (2013) Understanding and targeting cancer stem cells: therapeutic implications and challenges. Acta Pharmacol Sin 34(6): 732-740.

26. Deonarain MP, Christina A Kousparou, Agamemnon A Epenetos (2009) Antibodies targeting cancer stem cells: a new paradigm in immunotherapy?, MAbs 1(1): 12-25.

27. Zinzi L, Marialessandra Contino, Mariangela Cantore, Elena Capparelli, Marcello Leopoldo, et al. (2014) ABC transporters in CSCs membranes as a novel target for treating tumor relapse. Front Pharmacol 5: 163.

28. Morrison R, Stephen M Schleicher, Yunguang Sun, Kenneth J Niermann, Sungjune Kim, et al. (2011) Targeting the mechanisms of resistance to chemotherapy and radiotherapy with the cancer stem cell hypothesis. J Oncol.

29. Vaz AP, Moorthy P Ponnusamy, Surinder K Batra (2013) Cancer stem cells and therapeutic targets: an emerging field for cancer treatment. Drug Deliv Transl Res 3(2): 113-120.
30.Suman B Mondal, SG, Nan Zhu, Rongguang Liang, Viktor Gruev, et al. (2014) Real-time fluourescence image-guided onclology surgery. Adv Cancer Res 124: 171-211.

31. Vahrmeijer AL, HM, van der Vorst JR, van de Velde CJ, Frangioni JV (2013) Image-guided cancer surgery using near-infrared fluorescence. Nat Rev Clin Oncol 10(9): 507-518.

32. Gioux S, Hak Soo Choi, John V Frangioni (2010) Image-guided surgery using invisible near-infrared light: fundamentals of clinical translation. Mol Imaging 9(5): 237-255.

33. Butte PV, MA, Parrish Novak J (2014) Near-infrared imaging of brain tumors using the tumor paint BLZ-100 to achieve near-complete resection of brain tumors. Neurosurg Focus 36(2): E1.

34. Metildi CA, KS, Luiken GA, Talamini MA, Hoffman RM, et al. (2014) Fluorescently labeled chimeric anti-CEA antibody improves detection and resection of human colon cancer in a patient-derived orthotopic xenograft (PDOX) nude mouse model. J Surg Oncol 109(5): 451-458.

35. van Dam GM, Themelis G, Crane AML (2011) Intraoperative tumorspecific fluorescence imaging in ovarian cancer by folate receptor-alpha targeting: first in-human results. Nat Med 17(10): 1315-1319.

36. Aldave G, TS, Pay E, Marigil M, Bejarano B, et al. (2013) Prognostic value of residual fluorescent tissue in glioblastoma patients after gross total resection in 5-aminolevulinic Acid-guided surgery. Neurosurgery 72(6): 915-920. 